\title{
LIMIT THEOREMS RELATED TO A CLASS OF OPERATOR-SELF-SIMILAR PROCESSES
}

\author{
MAKOTO MAEJIMA
}

\section{Introduction and results}

An $\mathbf{R}^{d}$-valued $(d \geq 1)$ stochastic process $X=\{X(t)\}_{t \geq 0}$ is said to be operator-self-similar if there exists a linear operator $D$ on $\mathbf{R}^{d}$ such that for each $c>0$

$$
\left\{X(c t) \stackrel{\text { f.d. }}{=}\left\{c^{D} X(t)\right\}\right.
$$

where $\stackrel{f . d .}{=}$ means the equality for all finite-dimensional distributions and

$$
c^{D}=\exp \{(\ln c) D\}=\sum_{k=0}^{\infty} \frac{1}{k !}(\ln c)^{k} D^{k} .
$$

We refer the reader to $[\mathrm{HM} 1],[\mathrm{Sa}]$ and $[\mathrm{MM}]$ for more information about operator-self-similar processes. In the present paper, we show limit theorems related to a class of operator-self-similar processes, as a direct extension of [KS].

A probability distribution $\mu$ on $\mathbf{R}^{d}$ is said to be full if $\mu$ is not concentrated on a proper hyperplane and a full distribution $\mu$ on $\mathbf{R}^{d}$ is called operator-stable if it is infinitely divisible and there exist an invertible linear operator $B$ on $\mathbf{R}^{d}$ and a function $b:(0, \infty) \rightarrow \mathbf{R}^{d}$ such that for all $t>0$,

$$
\varphi(\theta)^{t}=\varphi\left(t^{B^{*}} \theta\right) e^{i b(t)}, \quad \theta \in \mathbf{R}^{d}
$$

where $\varphi$ is the characteristic function of $\mu, B^{*}$ is the adjoint operator of $B . \mu$ is called strictly operator-stable if we can choose $b(t) \equiv 0$. In this paper, we always assume $\mu$ is a full strictly operator-stable on $\mathbf{R}^{d}$. However, Sharpe ([Sh]) showed that if 1 is not an eigenvalue of $B$, then the operator-stable law can be centered so as to become strictly operator-stable. Thus the assumption for the strict operator-stability is not so restrictive. So, in the present paper, we always assume

Received May 13, 1994

Revised May 2. 1995. 


$$
\varphi(\theta)^{t}=\varphi\left(t^{B^{*}} \theta\right), \quad \theta \in \mathbf{R}^{d}
$$

The exponent $B$ is not necessarily unique. Let $\Lambda_{B}=\max \{\operatorname{Re} \sigma: \sigma \in \sigma(B)\}$ and $\lambda_{B}=\min \{\operatorname{Re} \sigma: \sigma \in \sigma(B)\}$, where $\sigma(B)$ is the set of all eigenvalues of $B$. Then it is known ([Sh]) that $\lambda_{B} \geq \frac{1}{2}$ and a full operator-stable measure $\mu$ can be classified as follows:

(i) $\mu$ is Gaussian. In this case, $B=\frac{1}{2} I$ can always be taken as an exponent of $\mu$.

(ii) $\mu$ is purely non-Gaussian. In this case, $\lambda_{B}>\frac{1}{2}$. When $\mu$ is $d$-dimensional $\alpha$-stable measure, we can take $B=\frac{1}{\alpha} I$.

(iii) $\mu$ is general. Theorem 1 in [HM2] allows us to consider the Gaussian component and the purely non-Gaussian component separately.

In this paper, we focus on purely non-Gaussian operator-stable laws, since Gaussian case $\left(B=\frac{1}{2} I\right)$ can be handled similarly to [KS]. The representation for the characteristic function of purely non-Gaussian operator-stable law with exponent $B$ is known as follows:

$$
\begin{aligned}
\varphi(\theta)=\exp \{i\langle\theta, c\rangle & \\
& \left.+\int_{S} \gamma(d x) \int_{0}^{\infty}\left[e^{i\left\langle\theta, s^{B} x\right\rangle}-1-i\left\langle\theta, s^{B} x\right\rangle I_{Q}\left(s^{B} x\right)\right] \frac{1}{s^{2}} d s\right\},
\end{aligned}
$$

where

$$
\begin{aligned}
& \theta \in \mathbf{R}^{d}, \quad c \in \mathbf{R}^{d}, \\
& S=\left\{x \in \mathbf{R}^{d}:\|x\|=1 \text { and }\left\|t^{B} x\right\|>1 \text { for all } t>1\right\}, \\
& Q=\left\{x \in \mathbf{R}^{d}:\|x\| \leq 1\right\} \\
& \gamma \text { is a probability measure on } S, \\
& \langle,\rangle \text { is the inner product in } \mathbf{R}^{d} .
\end{aligned}
$$

Let $Z_{B}$ be a purely non-Gaussian operator-stable random vector with exponent $B$ and let $\{\xi(k)\}_{k \in Z}$ be i.i.d. $\mathbf{R}^{d}$-valued random variables such that they belong to be domain of normal attraction of $Z_{B}$, namely

$$
n^{-B} \sum_{k=1}^{n} \xi(k) \stackrel{w}{\rightarrow} Z_{B}
$$


Let $\left\{S_{n}\right\}_{n=0}^{\infty}$ be an integer-valued random walk independent of $\{\xi(k)\}$ such that

$$
\frac{1}{n^{1 / \alpha}} S_{n} \stackrel{w}{\rightarrow} Z_{\alpha}
$$

where $Z_{\alpha}$ is one-dimensional $\alpha$-stable with $1<\alpha \leq 2$. In this paper, we are concerned with a sequence of dependent stationary random vectors $\left\{\xi\left(S_{k}\right)\right\}_{k=0}^{\infty}$ and study the asymptotic behavior of its cumulative sum

$$
W_{n}=\sum_{k=1}^{n} \xi\left(S_{k}\right)
$$

Kesten and Spitzer $([\mathrm{KS}])$ called this a random walk in random scenery when $d=1$, and proved that with a suitable normalization, $W_{[n t]}$ converges weakly to a self-similar process represented by a stable integral whose integrand is a local time.

To describe our theorem, we need some preliminaries. Let $\{Y(t)\}_{t \geq 0}$ be an $\alpha$-stable Lévy process with right continuous sample paths such that the distribution of $Y(1)$ is the same as that of $Z_{\alpha}$ in (4). Since $1<\alpha \leq 2, L_{t}(x)$, the local time of $Y(\cdot)$ at $x$, exists and we can take a version of $L_{t}(x)$ (denoted by $L_{t}(x)$ again) which is continuous in $(t, x)$. Let $\left\{Z_{B}(t)\right\}_{t \in \mathbf{R}}$ be an $\mathbf{R}^{d}$-valued Lévy process independent of $\{Y(t)\}_{t \geq 0}$ such that the distribution of $Z_{B}(1)$ is the same as that of $Z_{B}$ in (3). This $\left\{Z_{B}(t)\right\}$ is called an operator-stable Lévy process or operator-stable motion with exponent $B$. Each component $\left\{Z_{B}^{(i)}(t)\right\}, i=1,2, \cdots$, $d$, of $\left\{Z_{B}(t)\right\}$ is also a real-valued (not necessary stable) Lévy process. Hence the stochastic integral

$$
\Delta^{(i)}(t)=\int_{-\infty}^{\infty} L_{t}(x) d Z_{B}^{(i)}(x)
$$

can be defined for each $i$ as in [KS]. The $\mathbf{R}^{d}$-valued stochastic process whose $i$-th component is $\Delta^{(i)}(t)$ is denoted by

$$
\Delta(t)=\int_{-\infty}^{\infty} L_{t}(x) d Z_{B}(x),
$$

where $L_{t}(x)$ is a random scalar and $Z_{B}$ is a random vector.

Define $W_{t}$ for $t>0$ by

$$
W_{t}=W_{[t]}+(t-[t])\left(W_{[t]+1}-W_{[t]}\right),
$$

where $[t]$ is the integer part of $t$. Our theorems are the following. 
THeorem 1. Let $D=\left(1-\frac{1}{\alpha}\right) I+\frac{1}{\alpha} B$. Then any finite dimensional distribution of $\left\{n^{-D} W_{n t}\right\}_{t \geq 0}$ converges to that of $\{\Delta(t)\}_{t \geq 0} .\{\Delta(t)\}_{t \geq 0}$ is operator-selfsimilar with exponent $D$ and has stationary increments.

The latter half of Theorem 1 is easily seen by the definition of $\Delta(t)$.

THEOREM 2. $\left\{n^{-D} W_{n t}\right\}_{t \geq 0}$ converges weakly to $\{\Delta(t)\}_{t \geq 0}$ in the space $C\left([0, \infty): \mathbf{R}^{d}\right)$, provided that $\xi(0)$ is symmetric in the sense that $\xi(0) \stackrel{d}{=}$ $-\xi(0)$ when $\lambda_{B} \leq 1 \leq \Lambda_{B}$.

The idea of the proofs of these theorems is found in [KS]. The only technical difference in the proof of Theorem 1 comes from the fact that the characteristic function of operator-stable random vector (eq. (2)) does not have a simple form like that of one-dimensional stable random variable. This technical point can be dealt with the basic relation (1) and observations given in Lemmas 4 and 7 below. (Lemma 4 is trivial for the one-dimensional case.) The rest of the argument is exactly the same as in [KS].

For the proof of Theorem 2, we need some estimates for the "tail" behavior of the random vector belonging to the domain of normal attraction of operator-stable law. It will be recognized as in [W] that in the multidimensional case $P\left\{\left\|n^{-B} \xi\right\| \in\right.$ $A\}$ should be estimated instead of $P\{\|\xi\| \in A\}$. (See Lemmas 9, 11 and 12 below.) The estimates presented here can also be applied to a functional version of operator-stable limit theorem and other weak convergence theorem (see $[\mathrm{M}]$ ).

We give here a brief remark on the extra condition of the symmetricity of $\xi(0)$ for the case $\lambda_{B} \leq 1 \leq \Lambda_{B}$. When $d=1$, this case $\left(\lambda_{B}=\Lambda_{B}=1\right)$ corresponds to the so-called Cauchy case where the index of stability is 1 , and we often assume some conditions related to the symmetricity of $\xi(0)$. Such conditions are needed for the estimates for the tail behavior of random variables. However, the condition here is rather technical. The essential point would be whether 1 is an eigenvalue of $B$ or not. From this point of view, the extra condition in Theorem 2 might be weakened, although we do not try it in this paper.

We end this section with a remark about the $i$-th component $\Delta^{(i)}(t)$ of the $\mathbf{R}^{d}$-valued stochastic process $\Delta(t)$. If $B$ is diagonalizable over $\mathbf{R}$, then $Z_{B}^{(i)}(t)$ is one-dimensional stable $([\mathrm{H}])$. Thus $\Delta^{(t)}(t)$ is nothing but the process appearing in [KS]. Therefore it is self-similar. However if $B$ is not.semi-simple, then $Z_{B}^{(i)}(t)$ is not stable $([\mathrm{H}])$. Thus this process is not covered by $[\mathrm{KS}]$. If $B$ is not semi-simple, 
nor is $D$. Then it follows from Theorem 5.1 in $[\mathrm{M}]$ that $\Delta^{(i)}(t)$ is not self-similar. Therefore the $\mathbf{R}$-valued process $\Delta^{(i)}(t)$ is different from that in [KS].

\section{Proof of Theorem 1}

In the following, $\|\cdot\|$ stands for the ordinary Euclidean norm.

The first step of the proof is to represent $W_{n}$ as

$$
W_{n}=\sum_{k=0}^{n} \xi\left(S_{k}\right)=\sum_{u \in \mathbf{Z}} N_{n}(u) \xi(u),
$$

where $N_{n}(u)$ is the number of visits of the random walk $\left\{S_{n}\right\}$ to the point $u$ in the time interval $[0, n]$. All that are necessary about the occupation time $N_{n}(u)$ of $\left\{S_{n}\right\}$ and the local time $L_{t}(x)$ are found in [KS]. We collect some of them which we need later as lemmas. Consider the linear interpolation of $N_{n}(u)$ as $W_{t}$ as follows:

$$
N_{t}(u)=N_{[t]}(u)+(t-[t])\left(N_{[t]+1}(u)-N_{[t]}(u)\right) .
$$

For $-\infty<a<b<\infty$, define

$$
T_{t}^{n}(a, b)=\frac{1}{n} \sum_{n^{\frac{1}{\alpha}} a \leq u<n^{\frac{1}{\alpha} b}} N_{n t}(u)
$$

and

$$
\Gamma_{t}(a, b)=\int_{a}^{b} L_{t}(u) d u
$$

Lemma 1 ([KS]). For any $t_{1}, t_{2}, \cdots, t_{k} \geq 0$,

$$
\left\{T_{t_{j}}^{n}\left(a_{j}, b_{j}\right), 1 \leq j \leq k\right\} \stackrel{w}{\rightarrow}\left\{\Gamma_{t_{j}}\left(a_{j}, b_{j}\right), 1 \leq j \leq k\right\} .
$$

Lemma 2 ([KS]). For any $p \geq 1$,

$$
\sup _{u \in Z} E\left[N_{n}(u)^{p}\right]=O\left(n^{p\left(1-\frac{1}{\alpha}\right)}\right)
$$

and

$$
P\left\{N_{n}(u)>0 \text { for some } u \text { with }|u|>A n^{\frac{1}{\alpha}}\right\} \leq \varepsilon(A) \text { for } n \geq 1 \text {, }
$$

where $\varepsilon(A) \rightarrow 0$ as $A \rightarrow \infty$ and $\varepsilon(A)$ is independent of $n$.

In what follows, $C$ denotes an absolute constant which may differ from one 
inequality to another. Let $f=\log \varphi$, where $\varphi$ is the characteristic function of $Z_{B}$ defined in (2). We are going to show three lemmas.

Lemma 3 (The joint distribution of $\Delta(t)$ ). For any $t_{1}, t_{2}, \ldots, t_{k} \geq 0$ and $\theta_{1}$, $\theta_{2}, \ldots, \theta_{k} \in \mathbf{R}^{d}$

$$
E\left[\exp \left\{i \sum_{j=1}^{k}\left\langle\theta_{j}, \Delta\left(t_{j}\right)\right\rangle\right\}\right]=E\left[\exp \left\{\int_{-\infty}^{\infty} f\left(\sum_{j=1}^{k} L_{t_{j}}(u) \theta_{j}\right) d u\right\}\right] .
$$

Proof. The assertion easily follows from the facts that

$$
\int_{0}^{\infty} L_{t}(u) d Z_{B}(u)=\lim _{n \rightarrow \infty} \sum_{l=0}^{\infty} L_{t}\left(u_{l}^{n}\right)\left[Z_{B}\left(u_{l+1}^{n}\right)-Z_{B}\left(u_{l}^{n}\right)\right] \quad \text { w.p.1, }
$$

where $0=u_{0}^{n}<u_{1}^{n}<\cdots$ is a suitable sequance satisfying

$$
\lim _{l \rightarrow \infty} u_{l}^{n}=\infty, \quad \lim _{n \rightarrow \infty} \max _{l}\left(u_{l+1}^{n}-u_{l}^{n}\right)=0
$$

and that

$$
E\left[e^{i\left\langle\theta, Z_{B}\left(u_{l+1}^{n}\right)-Z_{B}\left(u_{l}^{n}\right)\right\rangle}\right]=\varphi(\theta)^{u_{l+1}^{n}-u_{l}^{n}},
$$

as in Lemma 5 in [KS].

Lemma 4. Let $\beta=1$ when $\Lambda_{B}<1$ and let $0<\beta<\frac{1}{\Lambda_{B}}$ when $\Lambda_{B} \geq 1$. Then for any $\theta_{1}$ and $\theta_{2} \in \mathbf{R}^{d}$, we have

$$
\left|f\left(\theta_{1}\right)-f\left(\theta_{2}\right)\right| \leq C\left\{\left\|\theta_{1}-\theta_{2}\right\|\left(1+\left\|\theta_{1}\right\|+\left\|\theta_{2}\right\|\right)+\left\|\theta_{1}-\theta_{2}\right\|^{\beta}\right\} .
$$

Proof. By (2),

$$
\begin{aligned}
& f\left(\theta_{1}\right)-f\left(\theta_{2}\right) \\
& =i\left\langle\theta_{1}-\theta_{2}, c\right\rangle \\
& \quad+\int_{S} r(d x) \int_{\left\{\left\|s^{B} x\right\| \leq 1\right\}}\left[e^{i\left\langle\theta_{1}, s^{B} x\right\rangle}-e^{i\left\langle\theta_{2}, s^{B} x\right\rangle}\right. \\
& \left.\quad-i\left\langle\theta_{1}-\theta_{2}, s^{B} x\right\rangle\right] \frac{1}{s^{2}} d s \\
& \quad+\int_{S} \gamma(d x) \int_{\left\{\left\|s^{B} x\right\|>1\right\}}\left[e^{i\left\langle\theta_{1}, s^{B} x\right\rangle}-e^{i\left\langle\theta_{2}, s^{B} x\right\rangle}\right] \frac{1}{s^{2}} d s .
\end{aligned}
$$

Observe that if $0<\beta \leq 1$, 


$$
\left|e^{i \xi_{1}}-e^{i \xi_{2}}\right| \leq 2^{(1-\beta) / \beta}\left|\xi_{1}-\xi_{2}\right|^{\beta} .
$$

For, if $\left|\xi_{1}-\xi_{2}\right| \geq 2^{1 / \beta}$, then $\left|e^{i \xi_{1}}-e^{i \xi_{2}}\right| \leq 2 \leq\left|\xi_{1}-\xi_{2}\right|^{\beta}$. If $\left|\xi_{1}-\xi_{2}\right|<2^{1 / \beta}$, then

$$
\left|e^{i \xi_{1}}-e^{i \xi_{2}}\right| \leq\left|\xi_{1}-\xi_{2}\right|=\left|\xi_{1}-\xi_{2}\right|^{1-\beta}\left|\xi_{1}-\xi_{2}\right|^{\beta} \leq 2^{(1-\beta) / \beta}\left|\xi_{1}-\xi_{2}\right|^{\beta} .
$$

Thus we have

$$
\begin{aligned}
\left|f\left(\theta_{1}\right)-f\left(\theta_{2}\right)\right| \leq & C\left\|\theta_{1}-\theta_{2}\right\| \\
& +2\left\|\theta_{1}-\theta_{2}\right\|\left(\left\|\theta_{1}\right\|+\left\|\theta_{2}\right\|\right) \int_{S} \gamma(d x) \int_{\left\{\left\|s^{B} x\right\| \leq 1\right\}} \frac{\left\|s^{B} x\right\|^{2}}{s^{2}} d s \\
& +2^{(1-\beta) / \beta}\left\|\theta_{1}-\theta_{2}\right\|^{\beta} \int_{S} \gamma(d x) \int_{\left\{\left\|s^{B} x\right\|>1\right\}} \frac{\left\|s^{B} x\right\|^{\beta}}{s^{2}} d s .
\end{aligned}
$$

Recall that $\lambda_{B}>\frac{1}{2}$ since $\mu$ is purely non-Gaussian operator-stable with exponent $B$. Hence

$$
\int_{S} \gamma(d x) \int_{\left\{\left\|s^{B} x\right\| \leq 1\right\}} \frac{\left\|s^{B} x\right\|^{2}}{s^{2}} d s<\infty .
$$

On the other hand, since $\beta<\frac{1}{\Lambda_{B}}$,

$$
\int_{S} \gamma(d x) \int_{\left\{\left\|s^{B} x\right\|>1\right\}} \frac{\left\|s^{B} x\right\|^{\beta}}{s^{2}} d s<\infty .
$$

Altogether we conclude the lemma.

Lemma 5. For any $t_{1}, t_{2}, \ldots, t_{k} \geq 0$ and $\theta_{1}, \theta_{2}, \ldots, \theta_{k} \in \mathbf{R}^{d}$,

$$
\sum_{u \in \mathbf{Z}} f\left(n^{-D^{*}} \sum_{j=1}^{k} N_{n t_{j}}(u) \theta_{j}\right) \stackrel{w}{\rightarrow} \int_{-\infty}^{\infty} f\left(\sum_{j=1}^{k} L_{t_{j}}(u) \theta_{j}\right) d u
$$

Proof. Since $n^{-D^{*}}=n^{-\left(1-\frac{1}{\alpha}\right)} n^{-\frac{1}{\alpha} B^{*}}$, we have, by the use of the relation (1),

$$
\begin{aligned}
\sum_{u \in \mathbf{Z}} f & \left(n^{-D^{*}} \sum_{j=1}^{k} N_{n t_{j}}(u) \theta_{j}\right) \\
& =\sum_{u \in \mathbf{Z}} \log \varphi\left(n^{-\left(1-\frac{1}{\alpha}\right)} \sum_{j=1}^{k} N_{n t_{j}}(u) n^{-\frac{1}{\alpha} B^{*}} \theta_{j}\right) \\
& =\sum_{u \in \mathbf{Z}} n^{-\frac{1}{\alpha}} \log \varphi\left(n^{-\left(1-\frac{1}{\alpha}\right)} \sum_{j=1}^{k} N_{n t_{j}}(u) \theta_{j}\right) .
\end{aligned}
$$


Thus it is enough to show that

$$
\sum_{u \in \mathbf{Z}} n^{-\frac{1}{\alpha}} f\left(n^{-\left(1-\frac{1}{\alpha}\right)} \sum_{j=1}^{k} N_{n t_{j}}(u) \theta_{j}\right) \stackrel{w}{\rightarrow} \int_{-\infty}^{\infty} f\left(\sum_{j=1}^{k} L_{t_{j}}(u) \theta_{j}\right) d u .
$$

The following argument is very similar to that in [KS]. For some small $\tau>0$ and large $M$, define

$$
\begin{aligned}
& A_{n, l}=\left\{u \in \mathbf{Z}: l \tau n^{\frac{1}{\alpha}} \leq u<(l+1) \tau n^{\frac{1}{\alpha}}\right\}, \quad l \in \mathbf{Z}, \\
& U(\tau, M, n)=\sum_{|u|>M \tau n^{\frac{1}{\alpha}}} n^{-\frac{1}{\alpha}} f\left(n^{-\left(1-\frac{1}{\alpha}\right)} \sum_{j=1}^{k} N_{n t_{j}}(u) \theta_{j}\right)
\end{aligned}
$$

and

$$
V(\tau, M, n)=\sum_{|l| \leq M}\left|A_{n, l}\right| n^{-\frac{1}{\alpha}} f\left(n^{-\left(1-\frac{1}{\alpha}\right)} \frac{1}{\tau n^{\frac{1}{\alpha}}} \sum_{y \in A_{n, l}} \sum_{j=1}^{k} N_{n t_{j}}(y) \theta_{\jmath}\right),
$$

where $\left|A_{n, l}\right|$ is the number of integers in $A_{n, l}$. Then

$$
\begin{gathered}
I:=\sum_{u \in \mathbf{Z}} n^{-\frac{1}{\alpha}} f\left(n^{-\left(1-\frac{1}{\alpha}\right)} \sum_{j=1}^{k} N_{n t_{j}}(u) \theta_{j}\right)-U(\tau, M, n)-V(\tau, M, n) \\
=\sum_{|l| \leq M} \sum_{u \in A_{n, l}} n^{-\frac{1}{\alpha}}\left\{f\left(n^{-\left(1-\frac{1}{\alpha}\right)} \sum_{j=1}^{k} N_{n t_{j}}(u) \theta_{j}\right)\right. \\
\left.-f\left(n^{-\left(1-\frac{1}{\alpha}\right)} \frac{1}{\tau n^{\frac{1}{\alpha}}} \sum_{y \in A_{n, l}} \sum_{j=1}^{k} N_{n t_{j}}(y) \theta_{j}\right)\right\} .
\end{gathered}
$$

Set, for a moment,

$$
g_{j}=N_{n t_{j}}(u) \quad \text { and } \quad h_{j}=\frac{1}{\tau n^{\frac{1}{\alpha}}} \sum_{y \in A_{n, l}} N_{n t_{j}}(y) .
$$

By Lemma 4,

$$
\begin{gathered}
E[|I|] \leq C(2 M+1)\left|A_{n, l}\right| n^{-\frac{1}{\alpha}} \sup _{u \in A_{n, l}}\left\{E \left[n^{-\left(1-\frac{1}{\alpha}\right)}\left\|\sum_{j=1}^{k}\left(g_{j}-h_{j}\right) \theta_{j}\right\|\right.\right. \\
\left.\left(1+n^{-\left(1-\frac{1}{\alpha}\right)}\left\|\sum_{j=1}^{k} g_{j} \theta_{j}\right\|+n^{-\left(1-\frac{1}{\alpha}\right)}\left\|\sum_{j=1}^{k} h_{j} \theta_{j}\right\|\right)\right] \\
\left.+E\left[n^{-\beta\left(1-\frac{1}{\alpha}\right)}\left\|\sum_{j=1}^{k}\left(g_{j}-h_{j}\right) \theta_{j}\right\|^{\beta}\right]\right\} \\
\leq C M \tau \sup _{u \in A_{n, l}}\left\{n^{-\left(1-\frac{1}{\alpha}\right)}\left(E\left[\left\|\sum_{j=1}^{k}\left(g_{j}-h_{j}\right) \theta_{j}\right\|^{2}\right]\right)^{1 / 2}\right. \\
\left(1+n^{-2\left(1-\frac{1}{\alpha}\right)} E\left[\left\|\sum_{j=1}^{k} g_{j} \theta_{j}\right\|^{2}\right]+n^{-2\left(1-\frac{1}{\alpha}\right)} E\left[\left\|\sum_{j=1}^{k} h_{j} \theta_{j}\right\|^{2}\right]\right)^{1 / 2}
\end{gathered}
$$




$$
\begin{gathered}
\left.+n^{-\beta\left(1-\frac{1}{\alpha}\right)}\left(E\left[\left\|\sum_{j=1}^{k}\left(g_{j}-h_{j}\right) \theta_{j}\right\|^{2}\right]\right)^{\beta / 2}\right\} \\
\leq C M \tau \sup _{u \in A_{n, l}}\left\{n^{-\left(1-\frac{1}{\alpha}\right)}\left(E\left[\| \sum_{j=1}^{k}\left(g_{j}-h_{j}\right)^{2}\right] \sum_{j=1}^{k}\left\|\theta_{j}\right\|^{2}\right)^{1 / 2}\right. \\
\left(1+n^{-2\left(1-\frac{1}{\alpha}\right)} E\left[\sum_{j=1}^{k} g_{j}^{2}\right] \sum_{j=1}^{k}\left\|\theta_{j}\right\|^{2}\right. \\
\left.+n^{-2\left(1-\frac{1}{\alpha}\right)} E\left[\sum_{j=1}^{k} h_{j}^{2}\right] \sum_{j=1}^{k}\left\|\theta_{j}\right\|^{2}\right)^{1 / 2} \\
\left.+n^{-\beta\left(1-\frac{1}{\alpha}\right)}\left(E\left[\sum_{j=1}^{k}\left(g_{j}-h_{j}\right)^{2}\right] \sum_{j=1}^{k}\left\|\theta_{j}\right\|^{2}\right)^{\beta / 2}\right\} .
\end{gathered}
$$

In $[\mathrm{KS}]$, it is proved that

$$
\sup _{u \in A_{n, l}} E\left[\left|g_{j}-h_{j}\right|^{2}\right] \leq C \tau^{\alpha-1} n^{2-\frac{2}{\alpha}} .
$$

Also by (6) in Lemma 2,

$$
\sup _{u \in \mathbf{Z}} E\left[N_{n}(u)^{2}\right]=O\left(n^{2-\frac{2}{\alpha}}\right)
$$

Hence we have

$$
E[|I|] \leq C M\left(\tau^{\frac{\alpha}{2}+\frac{1}{2}}+\tau^{1+\frac{\beta}{2}(\alpha-1)}\right)=C M \tau\left(\tau^{\frac{1}{2}(\alpha-1)}+\tau^{\frac{\beta}{2}(\alpha-1)}\right) .
$$

As to $U(\tau, M, n)$, as in [KS], we see that for large $n$ and for each $\eta>0$, we can take $M \tau$ so large that

$$
P\{U(\tau, M, n) \neq 0\} \leq \eta .
$$

Recall $\alpha>1$. Then take $\tau$ so small that

$$
C M \tau\left(\tau^{\frac{1}{2}(\alpha-1)}+\tau^{\frac{\beta}{2}(\alpha-1)}\right) \leq \eta^{2} .
$$

Then we can conclude that for such $\tau, M$ and large $n$,

$$
P\left\{\left|\sum_{u \in \mathbf{Z}} f\left(n^{-D^{*}} \sum_{j=1}^{k} N_{n t_{j}}(u) \theta_{j}\right)-V(\tau, M, n)\right|>\eta\right\} \leq 2 \eta .
$$

By the above consideration, it is enough to show the convergence of $V(\tau, M, n)$ in order to prove the lemma. By the use of the notation and the statement of Lemma 1 , we have

$$
V(\tau, M, n)=\sum_{|l| \leq M} \frac{\left|A_{n, l}\right|}{n^{\frac{1}{\alpha}}} f\left(\frac{1}{\tau} \sum_{j=1}^{k} T_{t_{j}}^{n}(l \tau,(l+1) \tau) \theta_{j}\right)
$$


which, as $n \rightarrow \infty$, weakly converges to

$$
\tau \sum_{|l| \leq M} f\left(\sum_{j=1}^{k} \frac{1}{\tau} \int_{l \tau}^{(l+1) \tau} L_{t_{j}}(y) d y \theta_{j}\right)
$$

where we have used $\frac{\left|A_{n, l}\right|}{n^{\frac{1}{\alpha}}} \rightarrow \tau$.

Finally, the continuity of $\sum_{j=1}^{k} L_{t_{j}}(u) \theta_{j}$ as a function of $u$ and the fact that $L_{t_{j}}(\cdot)$ has a.s. compact support imply that as $\tau \rightarrow 0$ and $M \rightarrow \infty,(10)$ converges to

$$
\int_{-\infty}^{\infty} f\left(\sum_{j=1}^{k} L_{t_{j}}(u) \theta_{j}\right) d u
$$

This together with (9) shows (8), completing the proof of the lemma.

We now return to the proof of the theorem. Denote the characteristic function of $\xi(u)$ by

$$
\lambda(\theta)=E\left[e^{i\langle\theta, \xi(u)\rangle}\right], \quad \theta \in \mathbf{R}^{d} .
$$

Then by (5)

$$
\begin{aligned}
I_{n} & :=E\left[\exp \left\{i \sum_{j=1}^{k}\left\langle\theta_{j}, n^{-D} W_{n t_{j}}\right\rangle\right\}\right] \\
& =E\left[\exp \left\{i \sum_{j=1}^{k}\left\langle\theta_{j}, n^{-D} \sum_{u \in \mathbf{Z}} N_{n t_{j}}(u) \xi(u)\right\rangle\right\}\right] \\
& =E\left[\prod_{u \in \mathbf{Z}} \lambda\left(n^{-D^{*}} \sum_{j=1}^{k} N_{n t_{j}}(u) \theta_{j}\right)\right] .
\end{aligned}
$$

We need more lemmas.

LEMMA 6.

$$
\lim _{n \rightarrow \infty} \sup _{u \in \mathbf{Z}} N_{n}(u) n^{-D^{*}} \theta=0 \text { in probability. }
$$

Proof. By (6) and (7) in Lemma 2, we have for some $p \geq 1$,

$$
\begin{aligned}
P\left\{\sup _{u \in \mathbf{Z}} N_{n}(u) \|\right. & \left.n^{-D^{*}} \theta \|>\eta\right\} \\
\leq P\left\{N_{n}(u)\right. & \left.>0 \text { for some } u \text { with }|u|>A n^{\frac{1}{\alpha}}\right\} \\
& +P\left\{\sup _{|u| \leq A n^{\frac{1}{\alpha}}} N_{n}(u)\left\|n^{-D^{*}} \theta\right\|>\eta\right\}
\end{aligned}
$$




$$
\begin{aligned}
& \leq \varepsilon(A)+\sum_{|u| \leq A n^{\frac{1}{\alpha}}} \frac{1}{\eta^{p}} E\left[N_{n}(u)^{p}\right]\left\|n^{-D^{*}} \theta\right\|^{p} \\
& =\varepsilon(A)+\sum_{|u| \leq A n^{\frac{1}{\alpha}}} \frac{1}{\eta^{p}} O\left(n^{p\left(1-\frac{1}{\alpha}\right)}\right) n^{-p\left(1-\frac{1}{\alpha}\right)}\left\|n^{-\frac{1}{\alpha} B^{*}} \theta\right\|^{p} \\
& =\varepsilon(A)+O\left(n^{\frac{1}{\alpha}}\left\|n^{-\frac{1}{\alpha} B^{*}} \theta\right\|^{p}\right) .
\end{aligned}
$$

Since for any $\varepsilon>0$,

$$
\left\|n^{-\frac{1}{\alpha} B^{*}}\right\| \leq C n^{-\frac{1}{\alpha}\left(\lambda_{B}-\varepsilon\right)}
$$

if we take $p$ such that $\frac{1}{\alpha}-\frac{1}{\alpha}\left(\lambda_{B}-\varepsilon\right) p<0$, the last term in (12) converges to 0 for fixed $\eta$ and $A$. If we next let $A$ tend to infinity, then the desired conclusion follows.

Lemma 7 (Lemma 6.1 of [MM]). Under (3), $\log \lambda(\theta) \sim \log \varphi(\theta)$ as $\theta \rightarrow 0$.

We now return to (11). By Lemmas 6 and 7,

$$
\begin{aligned}
\lim _{n \rightarrow \infty} I_{n} & =\lim _{n \rightarrow \infty} E\left[\prod_{u \in \mathbf{Z}} \varphi\left(n^{-D^{*}} \sum_{j=1}^{k} N_{n t_{j}}(u) \theta_{j}\right)\right] \\
& =\lim _{n \rightarrow \infty} E\left[\exp \left\{\sum_{u \in \mathbf{Z}} f\left(n^{-D^{*}} \sum_{j=1}^{k} N_{n t_{j}}(u) \theta_{j}\right)\right\}\right] \\
& =E\left[\exp \left\{\int_{-\infty}^{\infty} f\left(\sum_{j=1}^{k} L_{t_{j}}(u) \theta_{j}\right) d u\right\}\right] \text { (by Lemma 5) } \\
& =E\left[\exp \left\{i \sum_{j=1}^{k}\left\langle\theta_{j}, \Delta\left(t_{j}\right)\right\rangle\right\}\right] \text { (by Lemma 3). }
\end{aligned}
$$

The proof of Theorem 1 is thus completed.

\section{Proof of Theorem 2}

We prove the tightness of $\left\{n^{-D} W_{n t}\right\}$ by showing that for each $T>0$ and any $\eta>0$

$$
\lim _{n \rightarrow \infty} \lim _{\delta \downarrow 0} \sup _{\delta \downarrow} P\left\{\sup _{\substack{0 \leq t, s \leq T \\|t-s| \leq \delta}}\left\|\Delta_{t}^{n}-\Delta_{s}^{n}\right\| \geq \eta\right\}=0,
$$

where $\Delta_{t}^{n}=n^{-D} W_{n t}$. To this end, as in [KS], we first approximate $\Delta_{t}^{n}$ by $\bar{\Delta}_{t}^{n}$ plus a linear function $E_{n} t$ such that $\bar{\Delta}_{t}^{n}$ has the second moments, $E_{n}$ are bounded and 


$$
\underset{n \rightarrow \infty}{\lim \sup } P\left\{\sup _{t \leq T}\left\|\Delta_{t}^{n}-\bar{\Delta}_{t}^{n}-E_{n} t\right\| \geq \frac{1}{2} \eta\right\} \leq \frac{\varepsilon}{2},
$$

and then use Kolmogorov's moment criteria for $\bar{\Delta}_{t}^{n}$.

For any $\varepsilon>0$, choose large $A$ such that $\varepsilon\left(A T^{-\frac{1}{\alpha}}\right) \leq \frac{\varepsilon}{4}$, where $\varepsilon(\cdot)$ is the one defined in (7) in Lemma 2. Then we have

$$
\begin{aligned}
& P\left\{N_{n t}(u)>0 \text { for some }|u|>A n^{\frac{1}{\alpha}} \text { and } t \leq T\right\} \\
& \quad \leq P\left\{N_{n t}(u)>0 \text { for some }|u|>A n^{\frac{1}{\alpha}}\right\} \\
& \quad \leq \varepsilon\left(A T^{-\frac{1}{\alpha}}\right) \leq \frac{\varepsilon}{4} .
\end{aligned}
$$

We need several lemmas, where we always assume (3). For notational simplicity, we write $\xi$ for $\xi(0)$ in the following. Let

$$
\begin{gathered}
c_{n}(G)=n P\left\{\left\|n^{-B} \xi\right\| \in G\right\}, \quad G \in \mathfrak{B}((0, \infty)), \\
M(F)=\int_{S} \gamma(d x) \int_{0}^{\infty} I_{F}\left(s^{B} x\right) \frac{1}{s^{2}} d s, \quad F \in \mathfrak{B}\left(\mathbf{R}^{d} \backslash\{0\}\right)
\end{gathered}
$$

and

$$
c(G)=M(\{x:\|x\| \in G\}), \quad G \in \mathfrak{B}((0, \infty)) .
$$

Note that under (3), by the general central limit theorem for infinitely divisible laws in $\mathbf{R}^{d}$ (cf. Proposition 1.8.17 in [JM]),

$$
n P\left\{n^{-B} \xi \in F\right\} \rightarrow M(F)
$$

for every Borel set $F$ which is bounded away from the origin and $M(\partial F)=0$, and

$$
\lim _{\varepsilon \downarrow 0} \limsup _{n \rightarrow \infty} n \int_{\|x\|<\varepsilon}\langle\theta, x\rangle^{2} P\left\{n^{-B} \xi \in d x\right\}=0, \quad \theta \in \mathbf{R}^{d}
$$

(Recall that we are dealing with purely non-Gaussian case.) Assume for a moment that $\|\cdot\|$ is the "invariant norm" of [HJV]. In their norm, $c(\{y\})=0$ for each $y>0$. Then by eq. (7) in [W], we have

LEMMa 8. For every $y>0$,

$$
c_{n}([y, \infty)) \rightarrow c([y, \infty)) .
$$

Lemma 9. (i) Let $\rho>0$. Then 


$$
\sup _{n} \int_{0}^{\rho} y^{2} c_{n}(d y)<\infty
$$

(ii)

$$
\lim _{\varepsilon \downarrow 0} \limsup _{n \rightarrow \infty} \int_{0}^{\varepsilon} y^{2} c_{n}(d y)<\infty
$$

Proof. Suppose $\left\{\theta_{1}, \ldots, \theta_{d}\right\}$ is an orthonormal basis for $\mathbf{R}^{d}$. Then $\|x\|^{2}=$ $\sum_{i=1}^{d}\left\langle\theta_{i}, x\right\rangle^{2}$. Since

$$
\int_{0}^{\varepsilon} y^{2} c_{n}(d y)=n \int_{\|x\|<\varepsilon}\|x\|^{2} P\left\{n^{-B} \xi \in d x\right\}
$$

we conclude the lemma by (15) with $\theta=\theta_{1}, \ldots, \theta_{d}$.

Lemma 10 . Let $\rho>0$.

(i) If $\lambda_{B}>1$, then

$$
\int_{0}^{o} y c(d y)<\infty
$$

(ii) If $\Lambda_{B}<1$, then

$$
\int_{\rho}^{\infty} y c(d y)<\infty
$$

Proof. We have

$$
c((y, \infty))=M(\{x:\|x\|>y\})=\int_{S} \gamma(d x) \int_{0}^{\infty} I\left[\left\|s^{B} x\right\|>y\right] \frac{1}{s^{2}} d s .
$$

Note that for any $\delta>0$ there exists $C_{1}>0$ such that

$$
\left\|s^{B}\right\| \leq \begin{cases}C_{1} s^{\lambda_{B}-\delta} & \text { if } s \leq 1 \\ C_{1} s^{\Lambda_{B}+\delta} & \text { if } s>1\end{cases}
$$

By the use of (16), we have

$$
\begin{aligned}
c((y, \infty)) \leq & \int_{0}^{1} I\left[s>C_{2} y^{1 /\left(\lambda_{B}-\delta\right)}\right] \frac{1}{s^{2}} d s \\
& \quad+\int_{1}^{\infty} I\left[s>C_{2} y^{1 /\left(\Lambda_{B}+\delta\right)}\right] \frac{1}{s^{2}} d s \\
= & : I_{1}(y)+I_{2}(y),
\end{aligned}
$$


for some $C_{2}>0$.

(i) As $y \rightarrow 0, I_{2}(y)=O(1)$ and $I_{1}(y)=O\left(y^{-1 /\left(\lambda_{B}-\delta\right)}\right)$. If $\lambda_{B}>1$, we can find $\delta>0$ such that $1 /\left(\lambda_{B}-\delta\right)<1$. Thus $\int_{0}^{o} c((y, \infty)) d y<\infty$, which concludes (i).

(ii) As $y \rightarrow \infty, I_{1}(y)=o(1)$ and $I_{2}(y)=O\left(y^{-1 /\left(\Lambda_{B}+\delta\right)}\right)$. Thus, if $\Lambda_{B}<1$, we have $\int_{\rho}^{\infty} c((y, \infty)) d y<\infty$, concluding (ii).

Lemma 11. Let $\rho>0$. If $\lambda_{B}>1$, then

$$
\sup _{n} \int_{0}^{\rho} y c_{n}(d y)<\infty \text {. }
$$

Proof. It is obvious that for every $n \geq 1$

$$
\int_{0}^{\rho} y c_{n}(d y)<\infty
$$

and also

$$
\int_{0}^{o} y c(d y)<\infty
$$

by Lemma 10 (i). Note that $c_{n}(\cdot)$ and $c(\cdot)$ are Lévy measures on $(0, \rho)$, namely $\int_{0}^{\rho}\left(y^{2} \wedge 1\right) c_{n}(d y)<\infty$ and $\int_{0}^{\rho}\left(y^{2} \wedge 1\right) c(d y)<\infty$. Hence, by Lemmas 8 and 9 (ii), a convergence theorem of infinitely divisible laws (cf. Corollary 1.8.16 in [JM]) implies that the characteristic function

$$
f_{n}(\theta):=\exp \left\{\int_{0}^{\rho}\left(e^{i \theta y}-1\right) c_{n}(d y)\right\}, \quad \theta \in \mathbf{R}
$$

converges to

$$
f(\theta):=\exp \left\{\int_{0}^{\rho}\left(e^{i \theta y} \cdot 1\right) c(d y)\right\}, \quad \theta \in \mathbf{R}
$$

Thus

$$
\lim _{n \rightarrow \infty} \int_{0}^{\rho}\left(e^{\imath \theta y}-1\right) c(d y)
$$


exists. This together with Lemma 9 (i) concludes the lemma.

Lemma 12 . Let $\rho>0$. If $\Lambda_{B}<1$, then

$$
\sup _{n} \int_{\rho}^{\infty} y c_{n}(d y)<\infty .
$$

Proof. We first show the statement when $\xi$ is symmetric. Let $\varepsilon>0$, and choose $a$ so large that

$$
2 P\left\{\left\|n^{-B} \sum_{k=1}^{n} \xi(k)\right\|>a\right\}<\varepsilon \text { for all } n,
$$

which is possible by tightness, (see eq. (3)). Thus

$$
2 P\left\{\left\|n^{-B} \sum_{k=1}^{n} \xi(k)\right\|>y\right\}<\varepsilon \text { for all } y \geq a \text { and for all } n .
$$

Since $\{\xi(k)\}$ are symmetric, we have

$$
P\left\{\max _{1 \leq k \leq n}\left\|n^{-B} \xi(k)\right\|>y\right\} \leq 2 P\left\{\left\|n^{-B} \sum_{k=1}^{n} \xi(k)\right\|>y\right\} .
$$

Thus

$$
\begin{aligned}
{\left[P\left\{\left\|n^{-B} \xi\right\| \leq y\right\}\right]^{n} } & =P\left\{\max _{1 \leq k \leq n}\left\|n^{-B} \xi(k)\right\| \leq y\right\} \\
& =1-P\left\{\max _{1 \leq k \leq n}\left\|n^{-B} \xi(k)\right\|>y\right\} \\
& \leq 1-2 P\left\{\left\|n^{-B} \sum_{k=1}^{n} \xi(k)\right\|>y\right\}
\end{aligned}
$$

so that, for any $y \geq a$

$$
\begin{aligned}
n P\left\{\left\|n^{-B} \xi\right\|>y\right\} & \leq n\left\{1-\left[1-2 P\left\{\left\|n^{-B} \sum_{k=1}^{n} \xi(k)\right\|>y\right\}\right]^{1 / n}\right\} \\
& \leq \frac{2}{1-\varepsilon} P\left\{\left\|n^{-B} \sum_{k=1}^{n} \xi(k)\right\|>y\right\},
\end{aligned}
$$

since for a fixed $\varepsilon<1$,

$$
n\left\{1-(1-x)^{1 / n}\right\} \leq \frac{1}{1-\varepsilon} x, \quad \text { for any } 0 \leq x<\varepsilon .
$$


Hence

$$
\begin{aligned}
\sup _{n} \int_{a}^{\infty} n P\left\{\left\|n^{-B} \xi\right\|>y\right\} d y \\
\quad \leq \frac{2}{1-\varepsilon} \sup _{n} \int_{a}^{\infty} P\left\{\left\|n^{-B} \sum_{k=1}^{n} \xi(k)\right\|>y\right\} d y \\
\quad \leq \frac{2}{1-\varepsilon} \sup _{n} E\left[\left\|n^{-B} \sum_{k=1}^{n} \xi(k)\right\|\right] .
\end{aligned}
$$

By Theorem 3 in [HVW], if $\|\cdot\|$ is the ordinary Euclidean norm and $\Lambda_{B}<1$,

$$
E\left[\left\|n^{-B} \sum_{k=1}^{n} \xi(k)\right\|\right] \rightarrow E\left[\left\|Z_{B}\right\|\right]
$$

and hence

$$
\sup _{n} \int_{a}^{\infty} n P\left\{\left\|n^{-B} \xi\right\|>y\right\} d y<\infty
$$

for the "invariant norm" as well as for the ordinary Euclidean norm. This implies

$$
\sup _{n} \int_{a}^{\infty} y c_{n}(d y)<\infty
$$

On the other hand

$$
\int_{\rho}^{a} y c_{n}(d y) \rightarrow \int_{\rho}^{a} y c(d y)
$$

by Lemma 8 , thus we conclude

$$
\sup _{n} \int_{a}^{\infty} y c_{n}(d y)<\infty
$$

when $\xi$ is symmetric.

It remains to prove the lemma for the non-symmetric case and the following argument is a standard desymmetrization. For general $\xi$, let $\xi^{\prime}$ be an independent copy of $\xi$. Since $\xi-\xi^{\prime}$ is symmetric, we have shown

$$
\sup _{n} \int_{o}^{\infty} n P\left\{\left\|n^{-B}\left(\xi-\xi^{\prime}\right)\right\|>\frac{y}{2}\right\} d y=: K<\infty .
$$

Let 


$$
g_{n}(z)=\int_{\rho}^{\infty} n P\left\{\left\|n^{-B}(\xi-z)\right\|>\frac{y}{2}\right\} d y
$$

Then

$$
\sup _{n} E\left[g_{n}\left(\xi^{\prime}\right)\right]=K
$$

Let $b$ be so large that $P\{\|\xi\|>b\}<\frac{1}{2}$. Also let

$$
B_{b}=\left\{x \in \mathbf{R}^{d}:\|x\| \leq b\right\}
$$

and

$$
G_{n}=\left\{z \in \mathbf{R}^{d}: g_{n}(z) \leq 3 K\right\} .
$$

Then $B_{b}$ is not contained in $\mathbf{R}^{d} \backslash G_{n}$, because if it were, we would have

$$
\begin{aligned}
K & =\sup _{n} E\left[g_{n}\left(\xi^{\prime}\right)\right] \geq \sup _{n} E\left[g_{n}\left(\xi^{\prime}\right) I\left[\xi^{\prime} \in B_{b}\right]\right] \\
& >3 K E\left[I\left[\xi^{\prime} \in B_{b}\right]\right]=3 K P\{\|\xi\| \leq b\}>\frac{3}{2} K,
\end{aligned}
$$

which is impossible. Hence $B_{b} \cap G_{n} \neq \phi$. Let $z_{n} \in B_{b} \cap G_{n}$ for each $n \geq 1$. Since $\left\|z_{n}\right\| \leq b$, we have

$$
\int_{\rho}^{\infty} n P\left\{\left\|n^{-B} \xi\right\|>y\right\} d y \leq g_{n}\left(z_{n}\right)
$$

for large $n$. Since $g_{n}\left(z_{n}\right) \leq 3 K$, the proof is complete.

Remark. Lemmas 9-12 have been proved for the "invariant norm" of [HJV]. However, the compatibility of all norms on $\mathbf{R}^{d}$ implies the same conclusions for the ordinary Euclidean norm.

Lemma 13. If $\Lambda_{B}<1$, then $E[\|\xi\|]<\infty$ and $E[\xi]=0$.

Proof. The first part follows from Theorem 3 in [HVW]. The second part can be shown by the same way as in the one-dimensional case.

By Lemma 8 , we can find a $\rho$ such that for all large $n$

$$
\left(2 A n^{\frac{1}{\alpha}}+1\right) P\left\{\left\|n^{-\frac{1}{\alpha} B} \xi\right\|>\rho\right\} \leq \frac{\varepsilon}{4},
$$


for the "invariant norm". By the compatibility of all norms on $\mathbf{R}^{d}$ again, the same observation also follows for the ordinary Euclidean norm. In the following, once again the norm $\|\cdot\|$ stands for the ordinary Euclidean norm.

Set

$$
\begin{aligned}
\bar{\xi}(u) & =\xi(u) I\left[\left\|n^{-\frac{1}{\alpha} B} \xi(u)\right\| \leq \rho\right], \\
E_{n} & =n^{-D} E\left[\sum_{u \in \mathbf{Z}} N_{n}(u) \bar{\xi}(u)\right]
\end{aligned}
$$

and

$$
\bar{\Delta}_{t}^{n}=n^{-D} \sum_{u \in \mathbf{Z}} N_{n t}(u)\{\bar{\xi}(u)-E[\bar{\xi}(u)]\} .
$$

Again, for notational simplicity, we write $\bar{\xi}$ for $\bar{\xi}(0)$ in the following.

LEMMA 14. We have

$$
\left\|E\left[n^{-\frac{1}{\alpha} B} \bar{\xi}\right]\right\|=O\left(n^{-\frac{1}{\alpha}}\right),
$$

provided that $\xi$ is symmetric when $\lambda_{B} \leq 1 \leq \Lambda_{B}$.

Proof. When $\xi$ is symmetric, the left hand side of (18) is 0 . Hence it is enough to consider the case $\lambda_{B}>1$ or $\Lambda_{B}<1$.

When $\lambda_{\beta}>1$,

$$
\begin{aligned}
\sup _{n} n^{\frac{1}{\alpha}} \| & E\left[n^{-\frac{1}{\alpha} B} \bar{\xi}\right] \| \\
& =\sup _{n} n^{\frac{1}{\alpha}}\left\|E\left[n^{-\frac{1}{\alpha} B} \xi I\left[\left\|n^{-\frac{1}{\alpha} B} \xi\right\| \leq \rho\right]\right]\right\| \\
& \leq \sup _{n} \int_{0}^{o} y c_{n^{1 / \alpha}}(d y)<\infty
\end{aligned}
$$

by Lemma 11 .

When $\Lambda_{B}<1$, by the use of Lemmas 12 and 13 ,

$$
\begin{aligned}
\sup _{n} n^{\frac{1}{\alpha}} & \left\|E\left[n^{-\frac{1}{\alpha} B} \bar{\xi}\right]\right\| \\
& =\sup _{n} n^{\frac{1}{\alpha}}\left\|E\left[n^{-\frac{1}{\alpha} B} \xi I\left[\left\|n^{-\frac{1}{\alpha} B} \xi\right\| \leq \rho\right]\right]\right\| \\
& =\sup _{n} n^{\frac{1}{\alpha}}\left\|E\left[n^{-\frac{1}{\alpha} B} \xi I\left[\left\|n^{-\frac{1}{\alpha} B} \xi\right\|>\rho\right]\right]\right\| \\
& \leq \sup _{n} \int_{o}^{\infty} y c_{n^{1 / \alpha}}(d y)<\infty .
\end{aligned}
$$

This concludes the lemma. 
Let us return to the proof of Theorem 2 . We have by Lemma 14 ,

$$
\begin{aligned}
\left\|E_{n}\right\| & =\left\|n^{-\left(1-\frac{1}{\alpha}\right)} n^{-\frac{1}{\alpha} B} E\left[\sum_{u \in \mathbf{Z}} N_{n}(u) \bar{\xi}(u)\right]\right\| \\
& =\left\|n^{-\left(1-\frac{1}{\alpha}\right)} E\left[n^{-\frac{1}{\alpha} B} \bar{\xi}\right] E\left[\sum_{u \in \mathbf{Z}} N_{n}(u)\right]\right\| \\
& =n^{-\left(1-\frac{1}{\alpha}\right)} O\left(n^{-\frac{1}{\alpha}}\right)(n+1)=O(1) .
\end{aligned}
$$

We also have

$$
\begin{aligned}
& \Delta_{t}^{n}-\bar{\Delta}_{t}^{n}-E_{n} t \\
& =n^{-D} \sum_{u \in \mathbf{Z}} N_{n t}(u)[\xi(u)-(\bar{\xi}(u)-E[\bar{\xi}(u)])]-n^{-D} E\left[\sum_{u \in \mathbf{Z}} N_{n}(u) \bar{\xi}(u)\right] t \\
& =n^{-D} \sum_{u \in \mathbf{Z}} N_{n t}(u)[\xi(u)-\bar{\xi}(u)]+n^{-D} \sum_{u \in \mathbf{Z}} N_{n t}(u) E[\bar{\xi}(u)] \\
& \quad-n^{-D} E\left[\sum_{u \in \mathbf{Z}} N_{n}(u) \bar{\xi}(u)\right] t \\
& (19)=: n^{-D} \sum_{u \in \mathbf{Z}} N_{n t}(u)[\xi(u)-\bar{\xi}(u)]+Q_{n}(t),
\end{aligned}
$$

where by Lemma 14 for $t \leq T$,

$$
\begin{aligned}
\left\|Q_{n}(t)\right\| & =\left\|n^{-D} E[\bar{\xi}](n t+1-(n+1) t)\right\| \\
& \leq \operatorname{Tn}^{-\left(1-\frac{1}{\alpha}\right)}\left\|E\left[n^{-\frac{1}{\alpha} B} \bar{\xi}\right]\right\|=O\left(\frac{1}{n}\right) .
\end{aligned}
$$

It follows from (14) and (17) that

$$
\begin{aligned}
& P\left\{\sum_{u \in \mathbf{Z}} N_{n t}(u)[\xi(u)-\bar{\xi}(u)] \neq 0 \text { for some } t \leq T\right\} \\
& \leq P\left\{\xi(u) \neq \bar{\xi}(u) \text { for some }|u| \leq A n^{\frac{1}{\alpha}}\right\} \\
& \quad+P\left\{N_{n t}(u)>0 \text { for some }|u|>A n^{\frac{1}{\alpha}} \text { and } t \leq T\right\} \\
& \leq\left(2 A n^{\frac{1}{\alpha}}+1\right) P\left\{\left\|n^{-\frac{1}{\alpha} B} \xi\right\|>\rho\right\}+\frac{\varepsilon}{4} \leq \frac{\varepsilon}{2} .
\end{aligned}
$$

Hence by (19) for any $\eta>0$,

$$
\limsup _{n \rightarrow \infty} P\left\{\sup _{t \leq T}\left\|\Delta_{t}^{n}-\bar{\Delta}_{t}^{n}-E_{n} t\right\| \geq \frac{1}{2} \eta\right\} \leq \frac{\varepsilon}{2} .
$$

We finally show

$$
E\left[\left\|\bar{\Delta}_{t}^{n}-\bar{\Delta}_{s}^{n}\right\|^{2}\right] \leq C(t-s)^{2-\frac{1}{\alpha}}
$$


If we could show (21), the relation (13), with the respective replacements of $\Delta_{t}^{n}$ and $\eta$ by $\bar{\Delta}_{t}^{n}$ and $\frac{\eta}{2}$, would follow, and it together with (20) implies (13). We have

$$
\begin{aligned}
& E\left[\left\|\bar{\Delta}_{t}^{n}-\bar{\Delta}_{s}^{n}\right\|^{2}\right] \\
& \quad=E\left[\left\|n^{-D} \sum_{u \in \mathbf{Z}}\left(N_{n t}(u)-N_{n s}(u)\right)(\bar{\xi}(u)-E[\bar{\xi}(u)])\right\|^{2}\right] \\
& \quad=\sum_{u \in \mathbf{Z}} E\left[\left(N_{n t}(u)-N_{n s}(u)\right)^{2}\right] n^{-2\left(1-\frac{1}{\alpha}\right)} E\left[\left\|n^{-\frac{1}{\alpha} B}(\bar{\xi}(0)-E[\bar{\xi}(0)])\right\|^{2}\right] \\
& \quad \leq \sum_{u \in \mathbf{Z}} E\left[\left(N_{n t}(u)-N_{n s}(u)\right)^{2}\right] n^{-2\left(1-\frac{1}{\alpha}\right)} E\left[\left\|n^{-\frac{1}{\alpha} B} \bar{\xi}(0)\right\|^{2}\right],
\end{aligned}
$$

where

$$
\begin{aligned}
\sup _{n} n^{\frac{1}{\alpha}} E\left[\left\|n^{-\frac{1}{\alpha} B} \bar{\xi}\right\|^{2}\right] & =\sup _{n} n^{\frac{1}{\alpha}} E\left[\left\|n^{-\frac{1}{\alpha} B} \xi\right\|^{2} I\left[\left\|n^{-\frac{1}{\alpha} B} \xi\right\| \leq \rho\right]\right] \\
& =\sup _{n} \int_{0}^{\rho} y^{2} c_{n^{1 / \alpha}}(d y)<\infty
\end{aligned}
$$

by Lemma 9. On the other hand, Kesten and Spitzer ([KS]) showed

$$
\sum_{u \in \mathbf{Z}} E\left[\left(N_{n t}(u)-N_{n s}(u)\right)^{2}\right] \leq C[(t-s) n]^{2-\frac{1}{\alpha}} .
$$

Thus (21) is given from (22)-(24) and the proof is completed.

Acknowlegement. The author wishes to thank the referee for a very careful reading of the original manuscript.

\section{REFERENCES}

[HHV] Hahn, M. G., Hudson, W. N. and Veeh, J. A., Operator stable laws: Series representations and domain of normal attraction. J. Theor. Probab., 2 (1989), 3-35.

[H] Hudson, W. H., Operator-stable distributions and stable marginals, J. Multivar. Anal., 10 (1980), 26-37.

[HJV] Hudson, W. N., Jurek, Z. J. and Veeh, J. A., The symmetry group and exponents of operator stable probability measures, Ann. Probab., 14 (1986), 1014-1023.

[HM1] Hudson, W. N. and Mason, J. D., Operator-self-similar processes in a finitedimensional space, Trans. Amer. Math. Soc., 273 (1982), 281-297.

[HM2] Hudson, W. N. and Mason, J. D., Operator-stable laws, J. Multivar. Anal., 11 (1981), 434-447.

[HVW] Hudson, W. N., Veeh, J. A. and Weiner, D. C., Moments of distributions attracted to operator-stable laws, J. Multivar. Anal., 24 (1988), 1-10.

[JM] Jurek, Z. J. and Mason, J. D., Operator-Limit Distributions in Probability Theory, Wiley, 1993. 
[KS] Kesten, H. and Spitzer, F., A limit theorem related to a new class of self similar processes, Z. Wahrscheinlichkeitstheorie verw. Gebiete, 50 (1979), 5-25.

[M] Maejima, M., Operator-stable processes and operator fractional stable motions, Probab. Math. Statist., 15 (1995), 449-460.

[MM] Maejima, M. and Mason, J. D., Operator-self-similar stable processes, Stoch. Proc. Appl., 54 (1994). 139-163.

[Sa] Sato, K., Self-similar processes with independent increments, Probab. Th. Rel. Fields, 89 (1991), 285-300.

[Sh] Sharpe, M., Operator-stable probability distributions on vector groups, Trans. Amer. Math. Soc., 136 (1969), 51-65.

[W] Weiner, D. C., On the existence and convergence of pseudomoments for variables in the domain of attraction of an operator stable distribution, Proc. Amer. Math. Soc., 101 (1987), 521-529.

Department of Mathematics

Faculty of Science and Technology

Keio University

3-14-1, Hiyoshi, Kohoku-ku

Yokahama 223, Japan 